Fifth Meeting, 14th March 1890.

R. E. Allardict, Esq., M.A., Vice-President, in the Chair.

\title{
On the different possible non-linear arrangements of eight men on a Chess-board.
}

By T. B. Sprague, M.A.

The question having been proposed to me as a puzzle: To arrange eight men on a chess-board, so that no two of them shall be in the same line, - that is to say, that no two are to be in the same column, nor in the same row, nor in the same diagonal line,-I succeeded before very long in solving it by finding the annexed arrangement. (Fig. 45.)

Having been subsequently informed that other solutions had been found, I set to work systematically to ascertain how many there are, with the result that I found that there are twelve essentially different arrangements, and no more, which satisfy the required condition. The number is much larger, if we include the arrangements which are really the same, but are presented under a different aspect.

For instance, the above arrangement may be presented under eight aspects (Fig. 47). Here Nos. 2, 3 and 4 are got from No. 1 by turning the chess-board round clockwise, so that the side which was originally at the top becomes successively the right-hand side, the bottom side, and the left-hand side; each of the four sides of the board being uppermost in turn. No. 5 is got from No. 1 by inverting the position of the men on the board; and then Nos. 6, 7, 8 are got by turning it round as before.

It is clear that the number of arrangements which satisfy the condition that no two men shall be in the same column and no two in the same row, is 8.7.6.5.4.3.2; and if we assume that each essentially different arrangement (or, for brevity, each distinct arrangement) may be presented under eight aspects, we see that the total number of distinct arrangements which satisfy the condition, is 7.6.5.4.3.2, or 5040. The introduction of the further condition, that no two men are to be on the same diagonal line, reduces the number of arrangements to 12 ; but this is a conclusion 
I have arrived at by trial, and I have not been able to prove mathematically that 12 is the correct number. If we start by putting a man on the square marked $a$ in Fig. 46, then, by the conditions of the problem, the man in the second column cannot be placed on either of the three squares in it marked with a cross; and the man in the third column cannot be placed on either of the three squares in it similarly marked. We have thus five squares in the second column, on which the man can be placed; and if it is put on the square marked $b$, this prevents the man in the third column from being placed on either of the three squares marked with a circle; so that there are only two squares in the third column on which the man can be placed. If, however, we put the man on the square marked $c$ in the second column, we shall find that this leaves four squares open in the third column; while, if we place the man on any one of the three remaining squares in the second column, there will be three possible positions in the third column. We see thus that the number of possible positions in any column depends on the squares occupied in the preceding columns, in a manner which does not seem to admit of mathematical treatment.

In order to make an exhaustive examination of all the possible arrangements, it is not necessary to examine all the 5040 mentioned above. Before describing the process, it will be desirable to adopt a notation, by means of which we may indicate any square on the board, and any arrangement of men. I number the columns from left to right, and the rows from top to bottom, as shown in Fig. 45 ; then, in referring to any square, the number of the column is placed first and the number of the row, second; thus the spaces occupied by the men in Fig. 45 will be denoted by $(1,6),(2,1),(3,5),(4,2)$, $(5,8),(6,3),(7,7),(8,4)$ respectively. When we wish to indicate the whole arrangement, it is unnecessary to write down the numbers of the columns, as these all run in regular succession; and the arrangement in Fig. 45 is sufficiently denoted by (61528374). Turning now to Fig. 47, we see that the eight arrangements are denoted by the following numbers :-
(1) 61528374
(2) $57138642=i p(1)$
(3) $52617483=i r(1)$
(4) $75316824=r p(1)$
(5) $47382516=i(1)$
(6) $42861357=\operatorname{irp}(1)$
(7) $38471625=r(1)$
(8) $24683175=p(1)$

On examining these, we observe that (5) is got from (1) by 
inverting the order of the figures; and in the same way $(6)$ is got from (4), (7) from (3), and (8) from (2). This process I call inversion, and I denote it by the initial letter $i$, so that

$$
i(61528374)=47382516 \text {. }
$$

If we subtract each of the numbers in (1) from 9 , we get 38471625 , which is (7). This process I call reversion, and denote by $r$, so that

$$
r(61528374)=38471625 \text {. }
$$

Next, inverting the figures, we have

$$
\operatorname{ir}(61528374)=i(38471625)=52617483 \text {, }
$$

which is (3). We have by these processes got the arrangements $(5),(7),(3)$, from (1). In order to get the four remaining arrangements, we must interchange the columns and the rows. Thus in $(1),(1,6)$ is changed to $(6,1),(2,1)$ to $(1,2)$, and so on ; then arranging in the order of the new columns (or the old rows), we get $(1,2)$, $(2,4),(3,6),(4,8),(5,3),(6,1),(7,7)(8,5)$, or simply 24683175 , which is No. (8). This process I call perversion, and denote by $p$, so that

$$
p(61528374)=24683175 \text {. }
$$

Then inversion of $(8)$ gives us $(2)$; reversion of $(8)$ gives us $(4)$; and inversion of (4) gives us (6); these processes being symbolically denoted as follows : *

$$
\begin{aligned}
i p(61528374) & =i(24683175)=57138642, \\
r p(61528374) & =r(24683175)=75316824, \\
i r p(61528374) & =i(75316824)=42861357 .
\end{aligned}
$$

It will now be useful to show how, by means of the numbers, we

* It is not necessary for our present purposes to investigate the laws according to which our symbols of operation, $i, r, p$, combine with each otber; and $I$ will therefore content myself with stating a few of the principal laws, without any demonstration :-

$$
\begin{aligned}
& i^{2}=1, r^{2}=1, p^{2}=1 ; \\
& i r=r i, i p=p r, r p=p i ; \\
& i r p=r i p=i p i=r p r=p i r=p r i .
\end{aligned}
$$

As an illustration of the use of these relations, let $\mathrm{us}$ take the processes by which arrangement (2) in Fig. 47 is got from No. (1); that is to say, the process of turning the chess-board clock-wise through a quadrant. We have seen that $(2)=i p(1)$, so that the operation will be denoted by $i p$. If, now, we repeat this process, we have $(i p)^{2}=i p(i p)=i p \cdot p r=i p^{2} r=i r$; and this, as we have seen, is the process by which (3) is got from (1). Again, if we repeat the same process once more, we have

$$
(i p)^{3}=i p(i p)^{2}=i p, i r=r p r . r=r p r^{2}=r p ;
$$

and this, as we have seen, is the process by which (4) was got from (1). 
may ascertain, with regard to any particular arrangement, without any representation of it on a chess-board or in a diagram, whether it satisfies the condition that no two men are to be on the same diagonal line. For this purpose we need a sort of transformation of co-ordinates. If, instead of referring the position of each square to two sides of our board, as we have done hitherto, we refer it to one diagonal, AB (Fig. 48), and a perpendicular through its extremity, A, so as to fix the position of any square by the co-ordinates AM, PM, of its middle point, $P$, taking as our unit the half-diagonal of a square; then a square which is indicated by $(x y)$ according to the former plan, will be indicated by $(x+y-1, x-y)$, according to the latter plan; or, if we produce the diagonal backwards, and take as our new origin a point $O$, distant half a diagonal from the corner, the square formerly denoted by $(x y)$ will be now indicated simply by $(x+y, x-y)$.

According to this notation, the arrangement in Fig. 1 is indicated by $(7,-5),(3,1),(8,-2),(6,2),(13,-3),(9,3),(14,0),(12,4)$. Here the fact that all the eight numbers which stand first in the pairs, are different,-7, 3, 8,6,13,9, 14, 12,-shows that no two men are on the same diagonal line perpendicular to $A B$; and the fact that all the eight numbers which stand second in the pairs, are different, $-5,1,-2,2,-3,3,0,4,-$ shows that no two men stand on the same diagonal line parallel to $\mathrm{AB}$.

We see also that our problem may be stated without any mention of a chess-board: Required to arrange two sets of numbers, 1 - - 8, in eight groups, each containing one out of each set, so that the sums of the numbers in the eight groups shall all be different, and the differences of those numbers shall also all be different.

As another illustration, I will show how, by the same operations $i, r, p$, all the aspects in the text, instead of being got from No. (1), are got from one of the others, - say (4).

$$
\begin{gathered}
\text { We have }(4)=r p(1) \\
\therefore r(4)=r^{2} p(1)=p(1), \\
\text { and } p r(4)=p^{2}(1)=(1) ; \text { or }(1)=p r(4) .
\end{gathered}
$$

Then $(2)=i p(1)=i p p r(4)=i p^{2} r(4)=i r(4)$;

$(3)=i r(1)=i r p r(4)=i . i p i(4)=p i(4)$;

$(5)=i(1)=i p r(4)=i, i p(4)=p(4)$;

$(6)=i r p(1)=i r p p r(4)=i r p^{2} r(4)=i r^{2}(4)=i(4)$;

$(7)=r(1)=r p r(4)=r i p(4)$;

$(8)=p(1)=p p r(4)=r(4)$

It will be noticed that $(r p)^{-1}=p r$; and similarly $(i p)^{-1}=p i,(i r p)^{-1}=p r i=i r p$. 
I will now, by means of the preceding notation, explain the process of finding by trial the various possible arrangements. I begin by putting a man on the square $(1,1)$; then I put a man in the second column on the highest admissible square, which is $(2,3)$, because, by the conditions of the problem, the man must not be put on $(2,1)$ or $(2,2)$. In the third column, now, the man must not be put on $(3,1),(3,2),(3,3)$, or $(3,4)$ : I therefore put it on $(3,5)$. In the fourth column, the square $(4,2)$ is admissible, and I therefore put the man there; and the man in the fifth column is similarly put on $(5,4)$. Looking now at the position of the men on the board, we see that it is not possible, according to the conditions of the problem, to place a man anywhere in the sixth column. I therefore move on the last-placed man to the next admissible square, which is $(5,8)$; but it is still not possible to place a man in the sixth column. I therefore remove the man from the fifth column, and move on the man in the fourth column to the next admissible square, namely $(4,7)$. Then three more men may be placed, thus, $(5,2),(6,4)$, $(7,6)$; but it is not possible to place a man in the eighth column, and the last-placed men have therefore in succession to be moved on, and, when necessary, removed from the. board. This explanation will, I trust, be sufficient to enable my readers, with the chess-board before them, to understand the process by which the following arrangements were successively arrived at and found inadmissible, until the last was arrived at, namely, 15863724 .

\begin{tabular}{|c|c|c|c|}
\hline $\begin{array}{l}13524 \times \\
8 \times \\
1357246 \times \\
\frac{4 \times}{1358246 \times} \times \\
4 \times x \\
136275 \times \\
136824 \times \\
55 \times \\
137248 \times\end{array}$ & $\begin{array}{l}13824 \times \\
7 \times \\
13862 \times \\
425 \times \\
14253 \times \\
88 \times \\
14273 \times \\
142837 \times \\
1463 \times \\
63 \times\end{array}$ & $\begin{array}{c}1468253 x \\
146835 \times \\
77 \times \\
1473625 \times \\
1473825 \times \\
14752 \times \\
82 \times \\
1483 \times \\
14852 \times\end{array}$ & $\begin{array}{r}1526374 \times \\
1528374 \times \\
-63 \times \\
157248 \times \\
-63 \times \\
1582473 \times \\
-736 \times \\
15863724\end{array}$ \\
\hline
\end{tabular}

We can prove that this satisfies the conditions, by transforming it as above explained. First writing it in full, we get 1,$1 ; 2,5 ; 3,8 ; 4,6$; 5,$3 ; 6,7 ; 7,2 ; 8,4$; which for our present purpose may be more compactly written $\left|\begin{array}{l|l|l|l|l|l|l|l|l|l}1 & 2 & 3 & 4 & 5 & 6 & 7 & 8 \\ 1 & 5 & 8 & 6 & 3 & 7 & 2 & 4\end{array}\right|$. Writing the transfor- 
mation in a similar way, we get $\left|\begin{array}{r|r|r|r|r|r|r|r}2 & 7 & 11 & 10 & 8 & 13 & 9 & 12 \\ 0 & -3 & -5 & -2 & 2 & -1 & 5 & 4\end{array}\right|$; and since the eight numbers in the upper line are all different, and the eight numbers in the lower line are also all different, we see that this is an arrangement which satisfies the conditions; or, briefly, a solution. Proceeding thus, we get the following twelve solutions :-

\begin{tabular}{rlll|llll|rr}
15863724 &. &. & $(1)$ & 25741863 &. &. & $(5)$ & 27581463 &.$(9)$ \\
16837425 &. &. & $(2)$ & 26174835 &. &. & $(6)$ & $* 35281746$ &.$(10)$ \\
24683175 &. &. & $(3)$ & 26831475 &. &. & $(7)$ & 35841726 &.$(11)$ \\
25713864 &. &. & $(4)$ & 27368514 &. &. & $(8)$ & 36258174 &.$(12)$
\end{tabular}

As the work proceeds, various methods of shortening it slightly, suggest themselves; thus, if, when five columns, and consequently five rows, are occupied, the remaining three rows are adjacent rows, as, for instance, the fifth, sixth, and seventh; the problem is reduced to placing three men on a small board containing three squares in each side; and it is easy to see that this is impossible under the conditions. We therefore know that we cannot complete the arrangement, and it is useless to proceed to place a man on the sixth column. The same is the case if the two last remaining rows are adjacent.

We have to be on the watch against recording an old solution which presents itself under a new aspect. In order to explain this more clearly, $l$ have given in the following table the numbers indicating four aspects of each of the twelve solutions.
\begin{tabular}{l|c} 
& \\
$(1)$ & $a$ \\
$(2)$ & 15863724 \\
$(3)$ & $\mathbf{2 4 6 8 3 7 4 2 5}$ \\
$(4)$ & 25713864 \\
$(5)$ & 25741863 \\
$(6)$ & 26174835 \\
$(7)$ & 26831475 \\
$(8)$ & 27368514 \\
$(9)$ & 27581463 \\
$(10)$ & $* 35281746$ \\
$(11)$ & 35841726 \\
$(12)$ & 36258174
\end{tabular}

\begin{tabular}{|c|} 
Reversion. \\
$b=r(a)$ \\
84136275 \\
83162574 \\
75316824 \\
74286135 \\
74258136 \\
73825164 \\
73168524 \\
72631485 \\
72418536 \\
$* 64718253$ \\
64158273 \\
63741825
\end{tabular}

$\mid \begin{gathered}\text { Perversion. } \\ c=p(a) \\ 17582463 \\ 1746853 \\ 61528374 \\ 41582736 \\ 51842736 \\ 31758246 \\ 51468273 \\ 71386425 \\ 51863724 \\ * 53172864 \\ 57142863 \\ 63184275\end{gathered}$

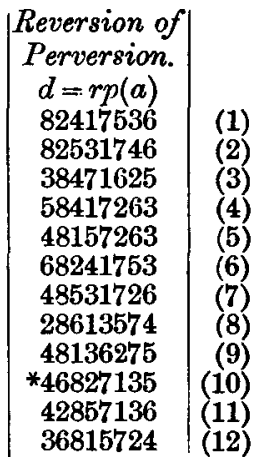

Here each aspect $b$ is got from the corresponding $a$ by reversion; each $c$ is got from $a$ by perversion; and each $d$ is got from $c$ by reversion. It is unnecessary to write down the remaining 
four aspects of each solution, as they will be got by simple inversion of the four that are here given; but it will be convenient to denote them by $\mathrm{A}, \mathrm{B}, \mathrm{C}, \mathrm{D}$, respectively, so that $\mathrm{A}=i(a)$, etc. This order is preferable in some respects to the order adopted in Fig. 47. We have placed (1) $a$ first because it is the first solution that our process gives us. It will be noticed that solution (10) differs from all the others, in that we get no new aspects by inverting the order of the numbers in it; thus if we invert the order in (10)a, we get 64718253 , which is $(10) b$; similarly the inversion of $(10) c$ gives us (10)d. As an example of an old solution presenting itself under a new aspect, we may take 17582463 , which the above table shows us is aspect $c$ of solution (1). Having arrived at this solution in the course of our process, we see by inspection of the board that it is a new aspect of an old solution; for such inspection informs us that it is an aspect of a solution commencing with 15 , which, therefore, we must have got already. It is easy to see further that, when in our trials we have got 17 as the commencing numbers, it is useless to make trial of arrangements that have a 2 in either the 3rd, 4th, 5th, or 6th place ; because, if any one of these should give us a solution, it must be another aspect of one that we have already got. For a similar reason, if we begin with 14 , it is useless to make trial of $142 \ldots$; if we begin with 15 , it is useless to make trial of $152 \ldots$ or of any arrangement containing 2 in the 4 th place ; if we begin with 16 , we similarly reject arrangements with 2 in the $3 \mathrm{rd}$, 4th, or 5 th place ; and when we begin with 18 , we reject arrangements which have 2 in the 3rd, 4th, 5th, 6th, or 7 th place.

There is no similar proposition when we begin with 2 ; but when we begin with 3 , it is useless to try arrangements beginning with 31 ; for by interchanging the columns and the rows, each of those arrangements must give us an arrangement commencing with 2 ; and all such we have already had. Similarly, if we began with 4, it would be useless to try any arrangement which has 1 in the 2 nd or 3rd column; if we began with 5 , it would be useless to try any arrangement which has 1 in the 2 nd, 3rd, or 4 th column; and so on.

The new aspects which we have thus far considered, are those which are contained in the column $c$, and are obtained by the process I have termed perversion. In order to recognize the others, we must first see whether there is a man nearer to a corner square than the one we started with. For instance, our process leads us to the solution 35714286 ; and as the square $(7,8)$ is next to a corner, this is 
a solution we have already got, and our table shows us that it is the $\mathrm{D}$ aspect of No. (6). Even if a solution contains no man nearer to a corner than the one we started with, it may still be a new aspect of an old solution: for instance, 36824175 contains 2 other men that are at the same distance from a corner as the one we started with, namely, in the third square; and the arrangements commencing with these respectively are 35841726 , which is No. (11); and 37285146 , which is the $B$ aspect of the same; while 36824175 is its $\mathrm{C}$ aspect. When we have completed all the trials that begin with 3, it is unnecessary to proceed any further; for, putting a man on the square $(1,4)$, we see at once that, if no man is to be nearer to a corner than this man is, the only admissible arrangement of the four men on the extreme columns and rows is the one shown in Fig. 49 ; and on trial we find that only one other man can be placed on the board consistently with the conditions of the problem.

As regards the remaining four squares in the first column, we see that by commencing with any one of them we shall simply get the $b$ aspects of the solutions we have already got; for instance, if we put a man on the 7 th square, we shall get the $b$ aspects of the solutions we got by putting the first man on the 2nd square.

On examining the twelve solutions we have got, the first thing that strikes us is the general absence of anything like symmetry, or apparent law, in them. It is, of course, clear that the conditions of the problem preclude bilateral symmetry; but we may have centric symmetry, so that to every man on the board there corresponds another at the same distance from the centre on the other side of it. One of our solutions is of this character, namely No. (10). In the numbers indicating it, 35281746, and 53172864, we observe that the sum of each pair of digits equidistant from the two ends, is 9 ; the 1st and the 8 th, the 2 nd and the 7 th, and so on. It results from this centric symmetry that, as already mentioned, there are only 4 aspects of this solution, and not 8 , as there are of the others. Hence the total number of arrangements which satisfy the conditions of the problem is $8 \times 11+4=92$; the total number of possible arrangements that contain one in each column and one in each row, being 8.7.6.5.4.3.2 $=40,320$.

From a study, either of the board or of the numbers in our table, we see that some of our solutions can be deduced the one from the other; for instance, if in No. (3) $a$ we remove 2 from the first place 
and put it in the last, we get 46831752 ," which represents No. (4)A. In order to see whether a transformation of this kind is possible we may proceed as follows, referring the men to a diagonal of the board and its perpendicular, as previously explained.

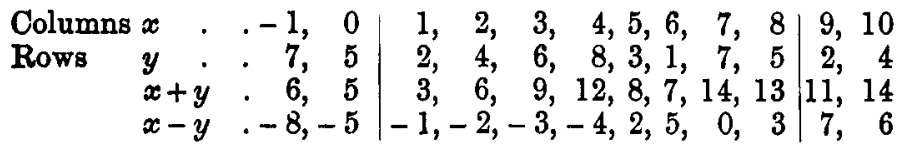

When we remove 2 from the first place to the last, and thus get 46831752 , the new values of $x+y$ and $x-y$ are 11 and 7 , which are different from any of the others; and this shows that the new arrangement is a solution. So, if we take 5 from the last place to the frst, $\dagger$ we get a solution 52468317, which is (8)C. But if we attempt to repeat the process in either direction, we get no new solution. Taking, for instance, 75246831, we have a value of $x+y$, namely 6 , which is the same as one of those we have already got; and this shows that the arrangement is not a solution. Similarly, if we take the arrangement 68317524 , we get a value of $x+y$, namely 14, which we have already got; and this arrangement also is not a solution. I indicate this connection between the three solutions graphically as follows:-

$$
\times-(8) \mathrm{C}-(3) a-(4) \mathrm{A}-\mathrm{x}
$$

Again, if we take the solution (11)c, namely 57142863, and subtract 1 from each of the numbers except 1 , which is to be replaced by 8 , we get 46831752 , which is (4)A. This operation I denote by $s$ (the initial letter of subtract), so that $46831752=s(57142863)$, and $(4 a)=i s(11 c)$. Each of these operations, $s$ and $t$, has a simple interpretation with reference to the arrangement of the men on the chessboard, which is so easily understood when the men are placed on the board, that it is unnecessary to explain it. I represent (1])c the connection between (11)c and (4)A graphically thus :- $\left.\right|_{(4) \mathrm{A}}$

It will be found that seven of our solutions, namely, Nos. (1), (2), (3), (4), (6), (8), and (11), can be deduced one from the other by

* This operation I denote by $t$ (the initial letter of trunspose), so that $46831752=t(24683175)$; whence $(4 A)=t(3 a)$.

+ This operation will be denoted by $t^{-1}$, so that $52468317=t^{-1}(24683175)$ and we have $(4 \mathrm{~A})=t(3 a)=t^{2}(\mathrm{SC})$. 
combinations of the 8 and $t$ operations; and I think the reader with the board before him will have no diffculty in understanding, without further explanation, the following diagram, which represents the connection between them.

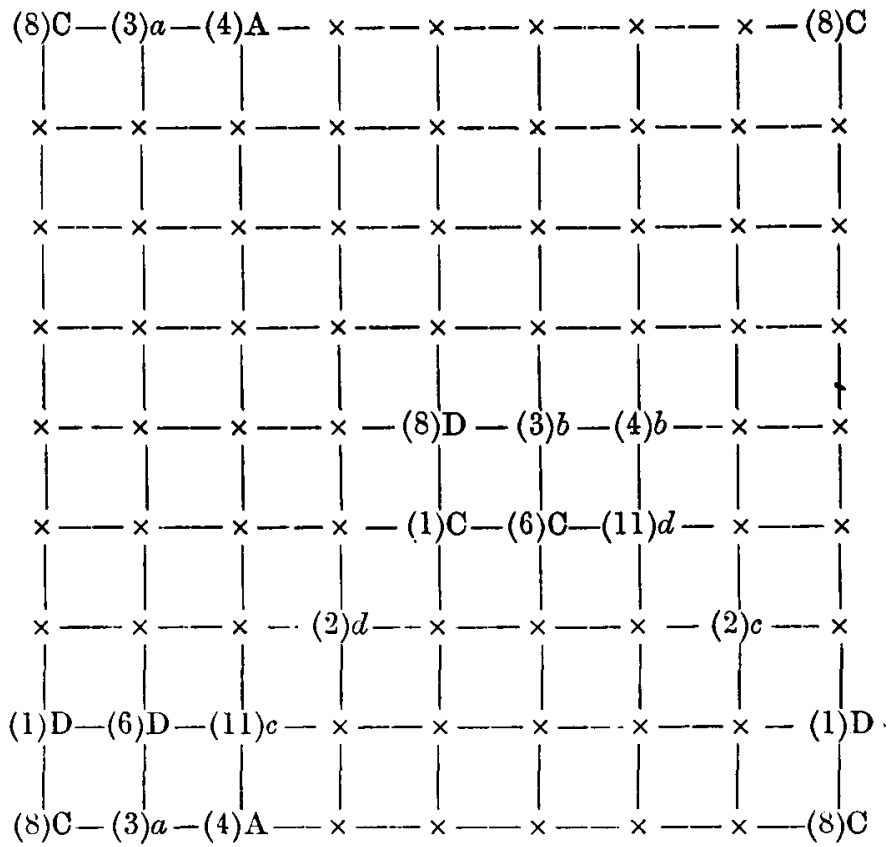

It will easily be seen that

$$
\begin{aligned}
(8 \mathrm{C})=s(1 \mathrm{D})=s^{2} t(2 c)=s^{11} t^{2}(11 d) & =s^{4} t^{2}(4 b) \\
& =s^{3} t^{3}(6 \mathrm{C}) \\
& =\text { etc. }
\end{aligned}
$$

The remaining 5 solutions, Nos. (5), (7), (9), (10), and (12), do not admit of being transformed in a similar way. It will be noticed that (2) $c$ and (2) $l$ are derived from the adjacent arrangements in a different way from the rest, namely, by the compound operation $s t$, or $s^{-1} t^{-1}$, or $s t^{-1}$, or $s^{-1} t$. Thus (2)c is got from (1)D by carrying a man from the right hand bottom corner of the bourd to the left hand top cornèr, and moving all the other men diagonally through one square downwards to the right. These two solutions have each a 
man in a corner square of the board, and I propose to call them "corner solutions".

Although I have satisfied myself by trial that there are 12 solutions and no more, I have not been able to discover any means of proving otherwise that this is the correct number. In order, if possible, to discover a law for the number of solutions, I have investigated the problem for the cases where the board has $4,5,6,7$, and 9 squares in its side; and the results are shown in the following table.

\begin{tabular}{|c|c|c|c|c|c|}
\hline $\begin{array}{c}\text { Number of } \\
\text { Sides. } \\
\text { (1) }\end{array}$ & $\begin{array}{c}\text { Distinct } \\
\text { Solutions. } \\
\text { (2) }\end{array}$ & $\begin{array}{c}\text { Of which } \\
\text { are Centric. } \\
(3)\end{array}$ & $\begin{array}{c}\text { Total } \\
\text { Solutions. } \\
(4)\end{array}$ & $\begin{array}{c}\text { Total } \\
\text { Arrangements. } \\
(5)\end{array}$ & $\begin{array}{c}\text { Quotient } \\
(5) \div(4)\end{array}$ \\
\hline 4 & 1 & 1 & 2 & 24 & 12 \\
5 & 2 & 1 & 10 & 120 & 12 \\
6 & 1 & 1 & 4 & 720 & 180 \\
7 & 6 & 2 & 40 & 5,040 & 126 \\
8 & 12 & 1 & 92 & 40,320 & $438 \frac{6}{2}$ \\
9 & 46 & 4 & 352 & 362,880 & $1030 \frac{10}{11}$ \\
\hline
\end{tabular}

There is no law visible in the progression of these numbers.

The solutions in the case of the boards containing $4,5,6$, and 7 squares in a side, are given in Fig. 50.

The solutions (1), (2), (4), (6) of the 7 -board can be derived one from the other in an endless series, as shown in the following scheme. Here $I$ have not thought it necessary to indicate the different aspects of each solution, but the 1 printed in heavy type is a different aspect from the 1 printed in ordinary type.

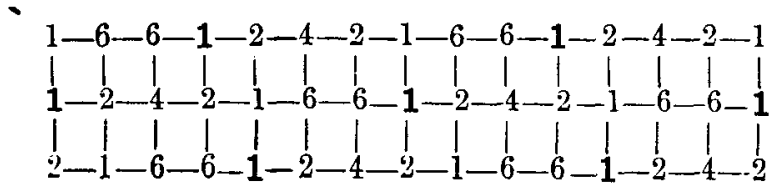

The succession of the numbers vertically is easily seen to be $1-2-6-4-6-2-1-1$.

The other solutions (3) and (5) canrot be similarly derived from each other or from any other.

The following are the solutions for the board which has 9 squares in each side. 

I. (1) 136824975
(2) -7285946
(3) -8692574
(4) 146392857
(5) -825397
(6) -7382596
(7) -925863
(8) -8397526
(9) 157938246
(10) - 42863
(11) -9642837
(12) 168374295
(13) 174835926
(14) $-\longrightarrow 9625$
II. (1) 241796358
(2) -7139685
(3) -8396157
(4) -9731685
(5) $-53168^{*}$
(6) 257936418
(7) -48136
(8) -8136974
(9) -96374
(10) -693147
(11) $-\longrightarrow-74$
(12) -9418637
(13) 261379485
(14) $--753948^{*}$
(15) -958473
(16) -3184975
(17) -9358417
(18) 275194683
(19) - 9631485
(20) 281479635
(21) -5396417
(22) -6931475
III. (1) 358296174
(2) --7146
(3) -9247186
(4) $362951847^{*}$
(5) $-8159247 *$
(6) --519724
(7) -9741825
(8) 372859164
(9) 386192574

IV. (1) 427918536

Of these solutions, the four marked with a star are centric, namely II.(5), II.(14), III.(4), and III.(5); and it may be useful to give the graphic representations of them ( $v$. Fig. 51).

In our search for a law, an obvious idea is to try whether any relation can be found to exist between the numbers of the solutions when the board has $n$ squares in its side and when it has $n+1$. When we examine from this point of view the solutions we have found, we inmediately see that it is only in exceptional cases that a solution in the one case can be deduced from one in the other case. If we take a solution for the board containing $(n+1)$ squares in its side, which for brevity we may call an $(n+1)$-board, and remove from the board one of the outside columns and one of the outside rows, we shall get an $n$-board, but we shall not in general get a solution; for, as there is a man on each column, and a man on each row, we shall have removed two men, unless it happens that there is a man on the corner square of the board which is common to the column and the row which we have removed. Conversely, when a solution for the $n$-board contains no man on one of the diagonals, we can get from this solution two solutions for the $(n+1)$-board, by producing the free diagonal, and adding a new column and a new row so as to intersect in the prolongation of the diagonal, first at the one end, and then at the other, and placing a new man on the corner square thus obtained. In this way solution (1) for the 5-board is got from 
the solution for the 4-board; solutions (1) and (2) for the 7-board are got from the solution for the 6-board; solutions (1) and (2) for the 8-board are got from solution (3) for the 7-board; and the following solutions for the 9-board are got from solutions for the 8-board :-

Nos. 1, 6, 10, 14 for the 9-board from No. 4 for the 8-board.

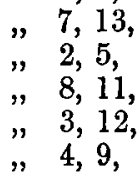

",

$\begin{array}{rrr} & 5 & \\ " & 6 & \\ " & 7 & \\ " & 9 & \end{array}$

By examining the diagrams for the solutions for the 9-board, it is easy to see that they will give us 32 solutions for the 10-board. The numbers of these "corner solutions" are-

$\begin{array}{cccc}\text { For the } & 4 \text {-board, none } \\ " & 5 & & 1 \\ " & 6 & " & \text { none } \\ " & 7 & " & 2 \\ " & 8 & " & 2 \\ " & 9 & " & 14 \\ " & 10 & " & 32\end{array}$

Here, again, there is no obvious law in the series of numbers.

It is sometimes possible to get a solution for the $n$-board from one for the $(n+1)$ board, by removing a man from the board, and both the column and the row containing it, and then closing up the board; for instance, solution (2) for the 5 -board gives in this way the solution for the 4-board. Conversely, one or more solutions for the $(n+1)$-board may sometimes be got by inserting a new column and a new row, and placing a man on the square in which they intersect. In this way we get:-

From Solution (1) for the 8-square, 1 solution for the 9-square.

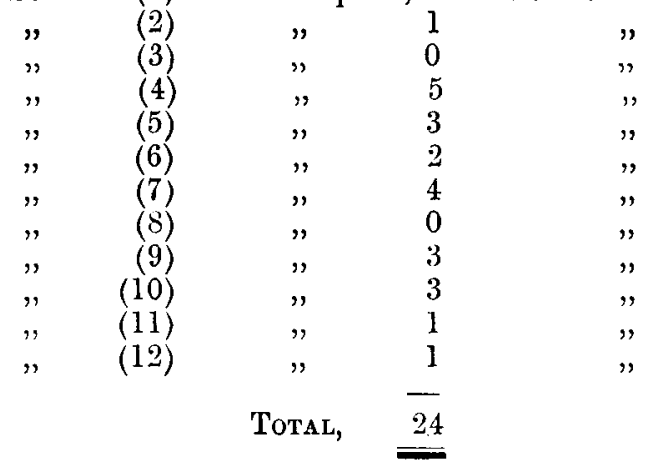


In consequence, however, of two of these solutions being given in 2 ways each, the actual solutions got are only 22 , or 8 in addition to the corner solutions. There are thus 24 solutions for the 9-board which cannot be got from solutions for the 8-board. This method of treating the question therefore does not lead us to a law.

A study of the diagrams suggests several interesting propositions for investigation; for instance, in no one of the solutions we have got for the 6-, 7-, 8-, and 9-boards, is there an arrangement of 4 men such as we have them in the 4-board solution; and $I$ am inclined to think it is impossible there should be, but I have not succeeded in proving it. Similarly, I am inclined to think it is impossible there should be an arrangement of 4 men on the outside columns and rows of a board, as shown in Fig. 52 ; but this also $I$ have not succeeded in proving.

On the equations of Vortex motion, with special reference to the use of polar co-ordinates.

By C. Chree, M.A., King's College, Cambridge.

In several previous communications* to the Society, I have considered the equations of vortex motion in two dimensions in a compressible fluid. In the present communication I propose to consider certain forms of the hydro-dynamical equations of a more general kind. In certain cases the fluid will be supposed to be rotating, prior to the introduction of the vortex motion, with uniform angular velocity about a fixed axis.

Using the same notation as in my previous papers, and supposing the axes of $x$ and $y$ rotating with uniform angular velocity $\omega$ about the axis of $z$, which is supposed fixed, we can easily prove by the method of my paper "On vortex motion in a rotating Fluid" $\dagger$ that the equations of three dimensional motion are the following-

$$
\begin{aligned}
\frac{\delta u}{\delta t}-2 \omega v-\omega^{2} x & =-\frac{1}{\rho} \frac{d p}{d x}+\mathrm{X} & \ldots & \ldots \\
\frac{\delta v}{\delta t}+2 \omega u-\omega^{2} y & =-\frac{1}{\rho} \frac{d p}{d y}+\mathrm{Y} & \ldots & \ldots \\
\frac{\delta w}{\delta t} & =-\frac{1}{\rho} \frac{d p}{d z}+Z & \ldots & \ldots
\end{aligned}
$$

* Proceedings, vol. V., p. 52 ; vol. VI., p. 59 ; vol. VII., p. 29. † Proceedings, vol. VII., p. 29. 